\title{
Tracing the origin of Treponema pallidum in China using next- generation sequencing
}

\author{
Jun Sun ${ }^{1, *}$, Zhefeng Meng ${ }^{2, *}$, Kaiqi Wu ${ }^{3}$, Biao Liu ${ }^{3}$, Sufang Zhang ${ }^{4}$ Yudan Liu ${ }^{4}$, \\ Yuezhu Wang ${ }^{5}$, Huajun Zheng ${ }^{5}$, Jian Huang ${ }^{5,6}$ and Pingyu Zhou ${ }^{1,4}$ \\ ${ }^{1}$ STD Institute, Shanghai Skin Disease Hospital, Shanghai, China \\ 2 Oncology Bioinformatics Center, Minhang Hospital, Fudan University, Shanghai, China \\ ${ }^{3}$ School of Laboratory Medicine, Wenzhou Medical University, Wenzhou, Zhejiang, China \\ ${ }^{4}$ Shanghai Skin Disease Hospital, Clinical School of Anhui Medical University, Shanghai, China \\ ${ }^{5}$ Shanghai-MOST Key Laboratory for Disease and Health Genomics, Chinese National Human Genome Center and National \\ Engineering Center for Biochip at Shanghai, Shanghai, China \\ ${ }^{6}$ Key Laboratory of Systems Biomedicine (Ministry of Education) and Collaborative Innovation Center of Systems Biomedicine, \\ Shanghai Center for Systems Biomedicine, Shanghai Jiao Tong University, Shanghai, China \\ * These authors have contributed equally to this work \\ Correspondence to: Pingyu Zhou, email: zpyls@yahoo.com \\ Jian Huang, email: jianhuang@sjtu.edu.cn
}

Keywords: Treponema pallidum, syphilis, NGS, SNVs, evolution, Pathology Section

Received: December 20,2015 Accepted: June 01,2016 Published: June 17, 2016

\section{ABSTRACT}

Syphilis is a systemic sexually transmitted disease caused by Treponema pallidum ssp. pallidum (TPA). The origin and genetic background of Chinese TPA strains remain unclear. We identified a total of 329 single-nucleotide variants (SNVs) in eight Chinese TPA strains using next-generation sequencing. All of the TPA strains were clustered into three lineages, and Chinese TPA strains were grouped in Lineage 2 based on phylogenetic analysis. The phylogeographical data showed that TPA strains originated earlier than did T. pallidum ssp. pertenue (TPE) and T. pallidum ssp. endemicum (TPN) strains and that Chinese TPA strains might be derived from recombination between Lineage 1 and Lineage 3 . Moreover, we found through a homology modeling analysis that a nonsynonymous substitution (I415F) in the PBP3 protein might affect the structural flexibility of PBP3 and the binding constant for substrates based on its possible association with penicillin resistance in $T$. pallidum. Our findings provide new insight into the molecular foundation of the evolutionary origin of TPA and support the development of novel diagnostic/therapeutic technology for syphilis.

\section{INTRODUCTION}

Treponema pallidum ssp. pallidum (T. pallidum ssp. pallidum; TPA) is the causative agent of syphilis [1] and infects more than 12 million people worldwide annually [2-4]. In China, although the elimination of syphilis was announced in the 1960s [5], the incidence of the disease has increased rapidly in recent years $[6,7]$. The number of reported syphilis cases increased more than 50-fold within 12 years, from 1993 to 2005 [8], and the latest Chinese national surveillance data of incidence rates for 2014 and 2013 were 419,091 and 406,772 cases, respectively (http://english.nhfpc.gov.cn/). Because syphilis cases were reported as early as the $16^{\text {th }}$ century in China [5], it is necessary to investigate the origin of the currently circulating TPA strains and to determine the transmission linkage between syphilis and global TPA strains.

Until recently, the genotyping of TPA strains relied on the characterization of the acidic repeat protein gene (arp), the tp0548 gene and the restriction fragment length polymorphism (RFLP) analysis of Treponema pallidum repeat gene (tpr) subfamily II $(t p r \mathrm{E}, t p r \mathrm{G}$, and $t p r \mathrm{~J})$, which was used to infer the prevalence and geographical distribution of TPA strains worldwide, the relationship 
Table 1: Background information and sequencing statistics for genome of T. pallidum.

\begin{tabular}{|l|l|l|l|l|l|l|l|l|l|l|l|}
\hline Strain & $\begin{array}{l}\text { Origin } \\
\text { Place }\end{array}$ & $\begin{array}{l}\text { Year of } \\
\text { isolation }\end{array}$ & Raw data & Clean data & Ratio & $\begin{array}{l}\text { unique } \\
\text { reads }\end{array}$ & $\begin{array}{l}\text { unique } \\
\text { ratio(\%) }\end{array}$ & $\begin{array}{l}\text { Coverage } \\
(\%)\end{array}$ & $\begin{array}{l}\text { Mean } \\
\text { depth }\end{array}$ & $\begin{array}{l}\text { SNV } \\
\text { number }\end{array}$ & $\begin{array}{l}\text { Count } \\
\text { treponemes }\end{array}$ \\
\hline SHC-0 & Shanghai & 2014 & 40416294 & 37469842 & $92.71 \%$ & 33483604 & $89.36 \%$ & $99.99 \%$ & 1899.73 & 286 & $1 \times 10^{7}$ \\
\hline SHD-R & Shanghai & 2014 & 28323906 & 26245436 & $92.66 \%$ & 21129166 & $80.51 \%$ & $99.99 \%$ & 2253.37 & 282 & $1 \times 10^{7}$ \\
\hline SHE-V & Shanghai & 2014 & 26352892 & 24296460 & $92.20 \%$ & 23352990 & $96.12 \%$ & $99.99 \%$ & 413.53 & 277 & $1 \times 10^{7}$ \\
\hline SHG-I2 & Shanghai & 2014 & 23141660 & 20869470 & $90.18 \%$ & 20312002 & $97.33 \%$ & $99.99 \%$ & 163.98 & 279 & $1 \times 10^{7}$ \\
\hline B3 & Shanghai & 2015 & 26527340 & 26340014 & $99.29 \%$ & 26046390 & $98.89 \%$ & $99.99 \%$ & 55.28 & 243 & $1 \times 10^{6}$ \\
\hline C3 & Shanghai & 2015 & 26746096 & 26581754 & $99.39 \%$ & 25883586 & $97.37 \%$ & $99.99 \%$ & 487.29 & 268 & $7 \times 10^{7}$ \\
\hline K3 & Shanxi & 2015 & 25504866 & 25293142 & $99.17 \%$ & 24091738 & $95.25 \%$ & $99.99 \%$ & 796.43 & 284 & $1 \times 10^{7}$ \\
\hline Q3 & Anhui & 2015 & 24819110 & 24649998 & $99.32 \%$ & 20028148 & $81.25 \%$ & $99.99 \%$ & 2047.53 & 281 & $1 \times 10^{7}$ \\
\hline
\end{tabular}

between strain types and the prevalence of neurosyphilis $[9,10]$. However, the $t p r$ gene family also displays wide genetic diversity among various subspecies of T. pallidum (syphilitic vs. non-syphilitic), supporting its critical roles in understanding bacterial evolution and immuneescaping mechanisms and in developing a vaccine against pathogenic treponemes [11]. To date, most TPA genomic sequence data are derived from American TPA strains. However, the genetic base underlying the evolution and emergence of Chinese TPA strains remain unclear.

As in many bacteria [12], whole-genome sequencing and phylogenetic linkages remain preferable for demonstrating the genetic relationships and molecular epidemiological characteristics of $T$. pallidum. Thus far, 12 full-genome sequences of treponemes have been published, including those of the TPA Nichols strain (GenBank: AE000520.1 and CP004010.2) [13, 14], TPA DAL-1 strain (GenBank: CP003115.1) [15], TPA Mexico A strain (GenBank: CP003064.1) [16], TPA Sea81-4 strain (GenBank: CP003679.1) [17], TPA SS14 strain (GenBank: CP004011.1 and CP000805.1) [13, 18], TPA Chicago strain (GenBank: CP001752.1) [19], T. pallidum ssp. pertenue (TPE) Samoa D strain (GenBank: CP002374.1) [20], TPE CDC-2 strain (GenBank: CP002375.1) [20], TPE Gauthier strain (GenBank: CP002376.1) [20], TPE Fribourg-Blanc strain (GenBank: CP003902.1) [21], T. pallidum ssp. endemicum (TPN) Bosnia A strain (GenBank: CP007548.1) [22], and T. paraluiscuniculi strain Cuniculi A (a rabbit treponemal isolate, GenBank: CP002103.1) [23]. These strains have contributed to the current understanding of the genetic features of treponemal species. As previously described [24-26], the members of the genus Treponema have high genetic similarity. Despite previous studies focusing on genetic variations in TPA or
TPE strains or other treponemes [20-22, 27-29], there is little evidence regarding the origin and dynamic evolution of global treponemal species. Notably, the theory of an Old World origin of venereal syphilis suggests that TPA originated in Europe [30, 31], while the New World hypothesis posits that TPA was introduced from America to Europe [32, 33]; thus, the origin or ancestry of TPA strains remains controversial and contradictory.

In the present study, we analyzed the genome sequences of TPA strains using next-generation sequencing technology to precisely define the genetic differences and origin of TPA in China.

\section{RESULTS}

\section{Sequenced genomes of eight Chinese TPA isolates}

To investigate the genetic variations present in TPA strains in China, eight DNA samples extracted from TPA-infected rabbit testes were sequenced using nextgeneration sequencing. A total of 20,869,470-37,469,842 high-quality reads per sample were determined (Table 1 , Supplementary Table 1). Of the unique reads, 95.61\% could be mapped to the reference genome (Nichols strain, GenBank: CP004010.2), and 99.99\% of the TPA genomic sequences were covered at a depth of at least 55 -fold in each sample (Table 1). The mean depth of coverage was approximately 1014-fold, and the greatest depth of coverage was 2253 -fold in the SHD-R sample (Table 1). The lowest depth of coverage was 55 -fold in the B3 sample, which may be associated with the lower number of treponemal cells in this sample (Table 1). 


\section{Genomic diversity of TPA strains in China and other countries}

To clarify the genetic diversity of Treponema, we obtained 11 treponemal genomic sequences from GenBank to analyze SNVs, including 6 TPA strains: Nichols, Chicago, Mexico A, SS14, DAL-1 and Sea81-4; 4 TPE strains: Gauthier, Samoa D, CDC2, and FribourgBlanc; and 1 TPN strain: Bosnia A. All of the genomic sequences were matched to the Nichols strain genome using Bowtie2, and the SNVs were subsequently annotated using SAMTools (Figure 1).

Treponemal species shared high similarity during their genetic evolution (genomic identity $>99.5 \%$ ) $[20,25,27]$. The number of SNVs found in individual Chinese strains ranged from 243 to 286 (median 280) and displayed $>70 \%$ similarity among the isolates. Fewer SNVs were identified in the genomes of the Chicago and DAL-1 strains compared to the Nichols strain (median 17 and 34, respectively). In contrast, the total number of
SNVs in the genomes of the SS14, Mexico A and Sea 81-4 strains ranged from 299 to 397 (median 324), representing the maximum number of SNVs compared to the Nichols strain among TPA subspecies. Furthermore, the number of SNVs in the TPE and TPN strains ranged from 1220 to 1467 (medium 1267), which was significantly higher than that in the TPA strains (Supplementary Table 2). The distinct pattern of SNVs identified among various TPA strains suggested distinct genetic relationships between these TPA strains. Therefore, we subsequently performed a genomic SNV-based phylogenetic analysis to determine the genetic linkage of these TPA strains.

As expected, all 14 of the TPA strains formed a large clade with a $100 \%$ bootstrap value in the maximumlikelihood tree, whereas five TPE and TPN strains formed another clade (Figure 2A, Supplementary Figure 1), suggesting that both of these groups of strains formed independent subspecies during evolution. Furthermore, three distinct clusters were observed within the TPA clade: Cluster 1 (Nichols, DAL-1, and Chicago), Cluster 2 (Chinese TPA, SHC-0, SHD-R, SHE-V, SHG-I2, B3,

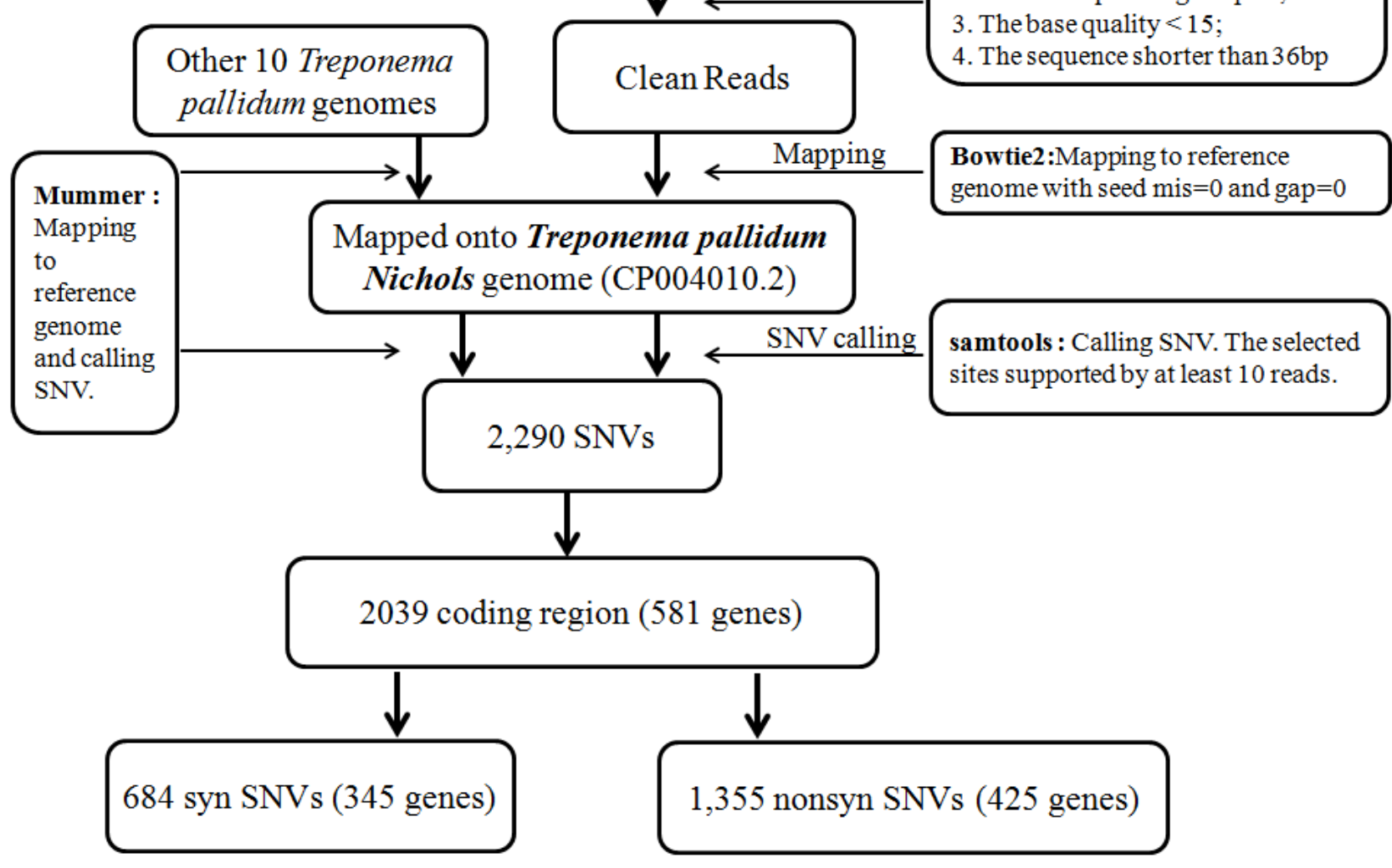

FASTX-Toolkits:

1. The sequence with ambiguous base

2. Cut the sequencing adapter;

3 . The base quality $<15$;

4. The sequence shorter than $36 \mathrm{bp}$

Bowtie2:Mapping to reference genome with seed mis $=0$ and gap $=0$

samtools : Calling SNV. The selected sites supported by at least 10 reads

Figure 1: Flowchart for analysis of the data derived from next-generation sequencing. 
Table2: Number of SNVs in the hree TPA lineages

\begin{tabular}{|l|l|l|l|}
\hline & Lineage 1 & Lineage 2 & Lineage 3 \\
\hline Tpr protein( $\boldsymbol{N = 7 1 )}$ & & & \\
\hline tprC & & & 11 \\
\hline tprD & & & 20 \\
\hline tprI & & 3 & 4 \\
\hline tprE & & 1 & \\
\hline tpr G & & 22 & \\
\hline tpr K & 4 & & \\
\hline tpr L & & & 6 \\
\hline $\begin{array}{l}\text { Hypothetical protein and putative } \\
\text { outer membrane protein }(\boldsymbol{N}=\mathbf{5 0})\end{array}$ & & & \\
\hline TPANIC_0136 & 18 & & 9 \\
\hline TPANIC_0548 & & 1 & 13 \\
\hline Hypothetical protein & 1 & 5 & 8 \\
\hline Intergenic $(\boldsymbol{N}=\mathbf{4 9})$ & & & 44 \\
\hline Other function( $\boldsymbol{N}=\mathbf{5 4})$ & & 4 & \\
\hline Penicillin-binding protein & & & \\
\hline $\begin{array}{c}\text { Methyl-accepting chemotaxis } \\
\text { protein (mcp2-1) }\end{array}$ & & 8 & 24 \\
\hline others & 7 & 44 & 11 \\
\hline Total & 30 & & 150 \\
\hline
\end{tabular}

C3, K3, and Q3) and Cluster 3 (Mexico A and SS14). Strain Sea81-4 was separated from these strains and was positioned more closely to Cluster 3 . Consistent with the observed SNV pattern among various treponemal species,
$\mathbf{A}$

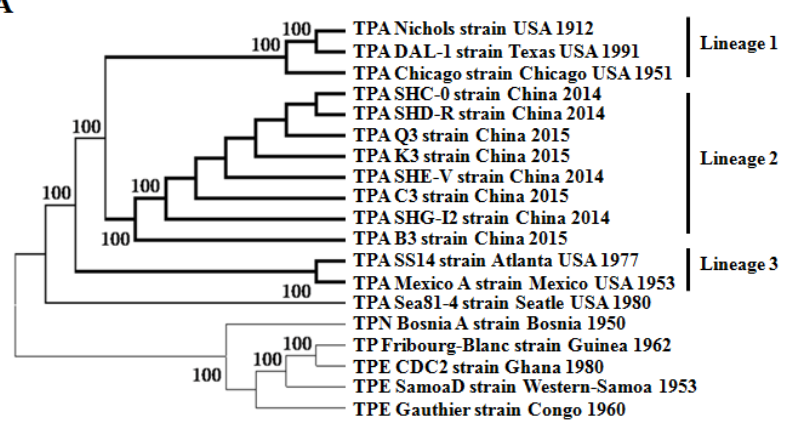

C

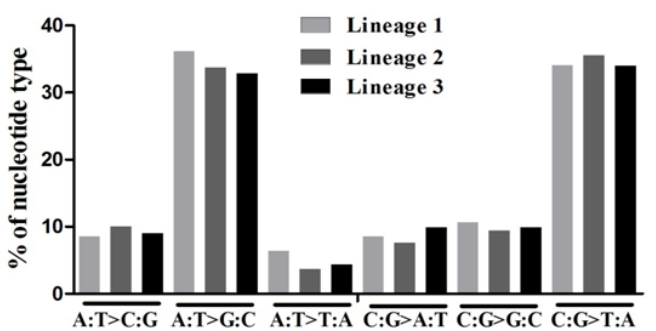

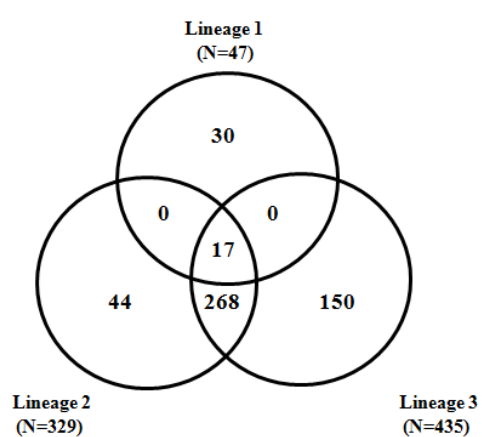

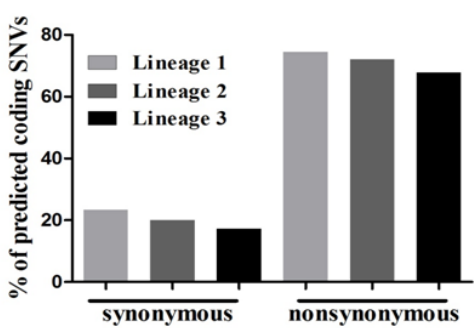

Figure 2: Characteristics of SNVs in different TPA lineages. A. Neighbor-joining phylogeny analysis of 697 SNVs defining TPA lineages. B. Venn diagram showing the number of SNVs in different TPA lineages. C. Percentage of mutation spectra of SNVs in different TPA lineages. D. Percentage of nonsynonymous and synonymous SNVs in different lineages. 
the strains with high-similarity SNV sites were clustered together. These results suggest that multiple independent lineages may have formed during the long-term evolution of TPA strains. To confirm this possibility, a Bayesian inferred tree of genomic SNVs was reconstructed using MrBayes 3.1 as previously described [34], and the results revealed the same phylogenetic relationships as did the ML tree (data not shown). Therefore, the three independent clusters were designated as three TPA lineages: Lineage 1 (Nichols, DAL-1, and Chicago), Lineage 2 (SHC0, SHD-R, SHE-V, SHG-I2, B3, C3, K3, and Q3), and Lineage 3 (Mexico A and SS14). In particular, Lineage 1 was closer to Lineage 2 than to Lineage 3 within the TPA strains in the genomic SNV-based ML tree (Figure 2A).

To investigate SNVs in various TPA lineages using a Venn diagram (Figure 2B), a total of 47, 329 and 435 SNVs were identified in the genomes from Lineage 1, Lineage 2, and Lineage 3, respectively. Lineage 1 did not share any common SNVs with Lineage 2 or with Lineage 3, whereas Lineage 2 and Lineage 3 shared 268 SNVs. Notably, more SNVs were shared between Lineage 2 and Lineage 3 than between Lineage 2 and Lineage 1 . However, Lineage 2 showed a closer genetic linkage to
Lineage 1 than to Lineage 3, as indicated by the genomic SNV-based ML tree (Figure 2A). Although further genetic analyses are required to interpret this phenomenon, it is possible that Lineage 3 shares some identity with treponemal strains in addition to TPA (e.g., TPE or TEN strains). Furthermore, a total of $224 \mathrm{SNVs}$ were designated as lineage-specific SNVs - 30 in Lineage 1, 44 in Lineage 2 and 150 in Lineage 3 (Figure 2B, Supplementary Table 3) - which were mainly distributed in the tpr gene family and encoded for hypothetical proteins or methyl-accepting chemotaxis proteins (mcp2-1) (Table 2). The tpr family genes experienced immune pressure in vivo and were employed for the evolutionary analysis and subtyping of TPA strains [35-37]. The distribution of lineage-specific SNVs also confirmed the critical role of $t p r$ family genes in the genetic evolution of TPA strains. In particular, Lineage 3 exhibited specific SNVs in genes encoding for methyl-accepting chemotaxis proteins (mcp2-1), which were previously identified from recombination events with TPE strains [16], suggesting a possible genetic linkage of the Mexico A strain with TPE strains.

Among the SNVs that were characterized in the three lineages, there was an obvious tendency of nucleotide
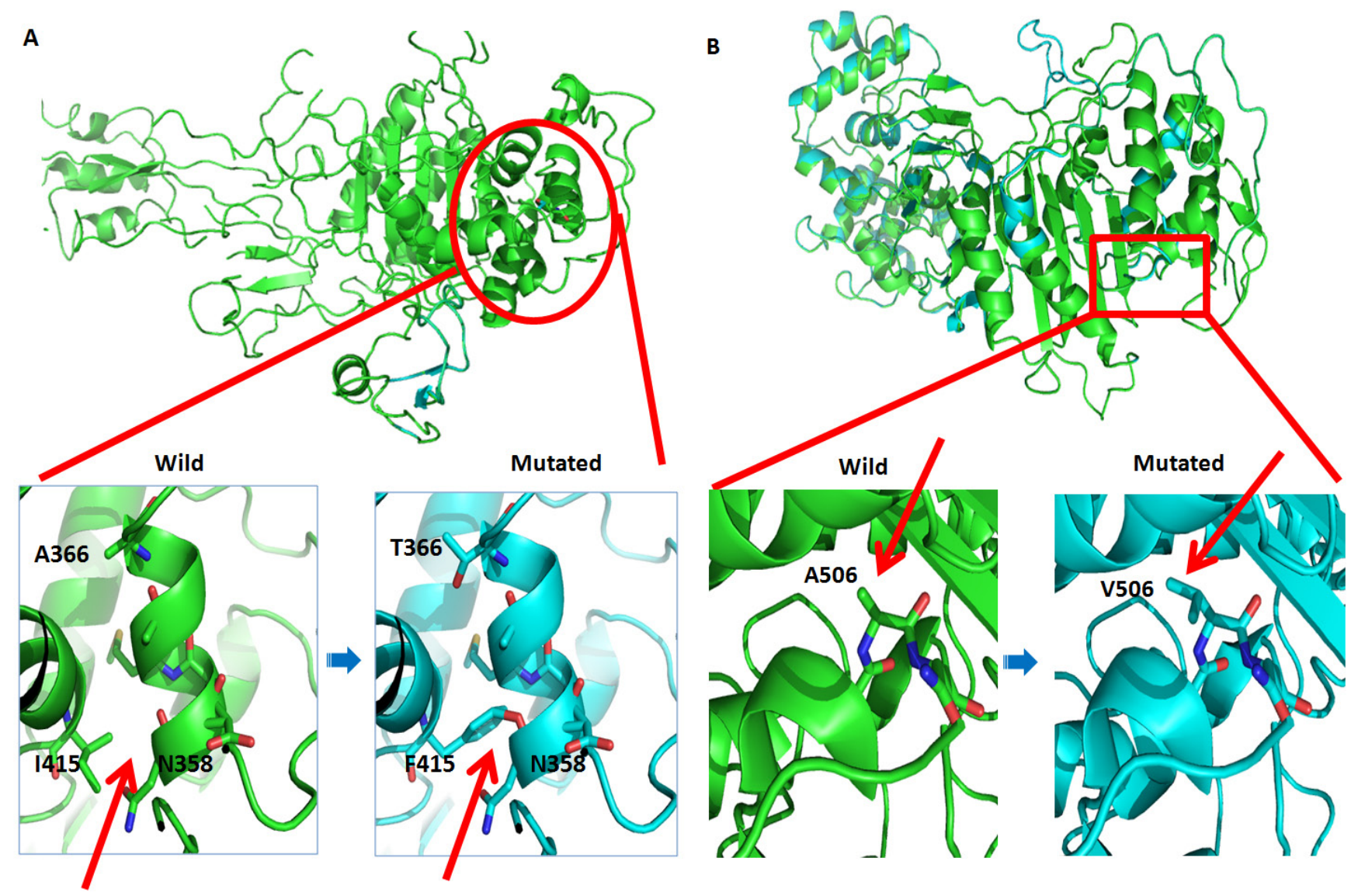

Figure 3: Predication of SNV effects on PBP3 and PBP2 proteins by homology model analysis. A. Ribbon representation of the PBP3 protein as predicted by the Phyre2 Server. Red arrows show the structural alteration caused by the SNVs compared to the wild-type model. B. Ribbon representation of the PBP2 protein as predicted by the Phyre2 Server. Red arrows show the structural alteration caused by the SNVs compared to the wild-type model. 
Table 3: The SNVs of penicillin regulatory Proteins in all Lineages.

\begin{tabular}{|c|c|c|c|c|c|c|c|}
\hline Gene & Annotation & SNV & Mutation & Mutation type & Lineage & Strain & $\begin{array}{l}\text { SIFT } \\
\text { Score }\end{array}$ \\
\hline TPANIC_0500 & $\begin{array}{l}\text { penicillin-binding } \\
\text { protein (pbp-1) }\end{array}$ & C537571T & P564L & nonsynonymous & 2,3 & $\begin{array}{l}\text { Strain C-0, D-R, } \\
\text { E-V, G-I2, B3, } \\
\text { C3, K3, Q3 and } \\
\text { SS14 }\end{array}$ & 0.2 \\
\hline \multirow[t]{2}{*}{ TPANIC_0547 } & $\begin{array}{l}\text { 4-hydroxy-3- } \\
\text { methylbut-2-enyl } \\
\text { diphosphate reductase/ } \\
\text { penicillin tolerance } \\
\text { protein (lytB) }\end{array}$ & T592290C & / & synonymous & 2,3 & $\begin{array}{l}\text { Strain } \\
\text { D-R, E-V, G-I2, } \\
\text { B3, C3, K3, } \\
\text { Q3, SS14 and } \\
\text { Mexico A }\end{array}$ & / \\
\hline & & T592614C & / & synonymous & 2,3 & 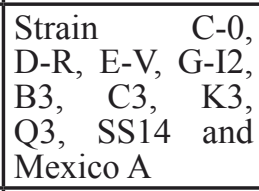 & / \\
\hline TPANIC_0705 & $\begin{array}{l}\text { penicillin-binding } \\
\text { protein (pbp-2) }\end{array}$ & G773571A & A506V & nonsynonymous & 2 & $\begin{array}{l}\text { Strain C-0, D-R, } \\
\text { E-V, G-I2, B3, } \\
\text { C3, K3 and Q3 }\end{array}$ & 0.03 \\
\hline \multirow[t]{3}{*}{ TPANIC_0760 } & $\begin{array}{l}\text { penicillin-binding } \\
\text { protein (pbp-3) }\end{array}$ & G824922A & A366T & nonsynonymous & 2 & $\begin{array}{l}\text { Strain C-0, and } \\
\text { B3 }\end{array}$ & 0.14 \\
\hline & & A825069T & $\mathrm{I} 415 \mathrm{~F}$ & nonsynonymous & 2 & $\begin{array}{l}\text { Strain C-0, D-R, } \\
\text { E-V, G-I2, B3, } \\
\text { C3 and Q3 }\end{array}$ & 0.03 \\
\hline & & $\mathrm{C} 825071 \mathrm{G}$ & $\mathrm{I} 415 \mathrm{M}$ & nonsynonymous & 2 & Strain K3 & 0.17 \\
\hline
\end{tabular}

a,functional SNVs predicted using SIFT (SIFT score $\leq 0.05$ as the deleterious effect).

mutations toward $\mathrm{C}: \mathrm{G}>\mathrm{T}: \mathrm{A}$ and $\mathrm{A}: \mathrm{T}>\mathrm{G}: \mathrm{C}$ in all of the lineages (Figure $2 \mathrm{C}$ ). Notably, the $\mathrm{C}: \mathrm{G}>\mathrm{G}: \mathrm{C}$ transversion was also significantly enriched in Lineage 1. Regarding the enrichment of either the $\mathrm{A}: \mathrm{T}>\mathrm{G}: \mathrm{C}$ or $\mathrm{C}: \mathrm{G}>\mathrm{T}: \mathrm{A}$ mutation, the GC content $(53 \%)$ of the genomes was not altered in all of the lineages, indicating that the biases of these transversions might not affect the usage of the codons and amino acids in TPA strains grown under different selective pressures [38, 39]. In addition, a higher proportion of nonsynonymous SNVs was displayed in all of the lineages (Figure 2D), potentially affecting the function of the respective gene-encoded products. To investigate these effects, we focused our analyses on nonsynonymous SNVs and found that 11 genes ( $\geq 2$ SNVs per gene) were most highly affected, some of which were potential membrane genes (TPANIC_0136; TPANIC_0326; TPANIC_0548; TPANIC_0865 and TPANIC_0515) or known virulence factors, such as tpr family genes (TPANIC_0897; TPANIC_0317; TPANIC_0313 and TPANIC_1031) and penicillin-binding protein (TPANIC_0760). Although the most highly affected genes were non-specific for unique lineages, Lineage 2 contained the maximum number of $t p r$ genes that were highly affected by SNVs. Given that the reference strain spread among European and US populations, these lineage-specific SNVs found in tpr family genes in the Chinese strains might reflect selective pressure in Chinese or Asian populations.

\section{Potential effects of SNVs on the response of syphilis patients to penicillin treatment}

Penicillin has been recommended worldwide as a first-line drug for the treatment of all stages of syphilis [2]. Although there is no evidence available for penicillin resistance in $T$. pallidum, penicillin treatment failure has caused concern [40-44]. In this study, we identified seven SNVs in penicillin-regulatory genes, including five nonsynonymous mutations in penicillin-binding proteins (PBPs) and two synonymous mutations in a penicillin-tolerance protein (lytB) (Table 3). Because a nucleotide mutation in endogenous PBPs might lead to resistance against $\beta$-lactams $[11,45]$, we subsequently predicted the effects of five nonsynonymous SNVs on the function of PBP genes in Lineage 2 and Lineage 3. Two nonsynonymous SNVs (G773571A, A506V, in PBP2 protein and A825069T, I415F, in PBP3 protein) in Chinese TPA strains were likely to impair the function of proteins as predicted through a SIFT analysis (Table 
3). A homology modeling analysis revealed that the I415 to F415 substitution altered the structure of side chains, which affected the structural flexibility of the PBP3 protein and might thereby change the binding constant for substrates of some proteins (Figure 3A). In contrast, the A506 to V506 mutation did not cause any structural alterations, although a deleterious effect was indicated in the SIFT analysis (Table 3, Figure 3B). These results suggest that the SNVs present in PBP genes might compromise the responses of syphilis patients to penicillin treatment, although more evidence is needed to confirm these results in the future.

\section{The SNVs validated by Sanger sequencing}

To validate the variants identified by NGS, we amplified and sequenced 13 genes and 3 intergenic regions covering $23 \mathrm{SNV}$ sites, including $6 \mathrm{SNVs}$ in the PBPs gene, $7 \mathrm{SNVs}$ in the $t p r$ genes and 10 randomly selected SNVs, using PCR-based Sanger sequencing technology (ABI 3730 DNA Analyzer, Applied Biosystems). The

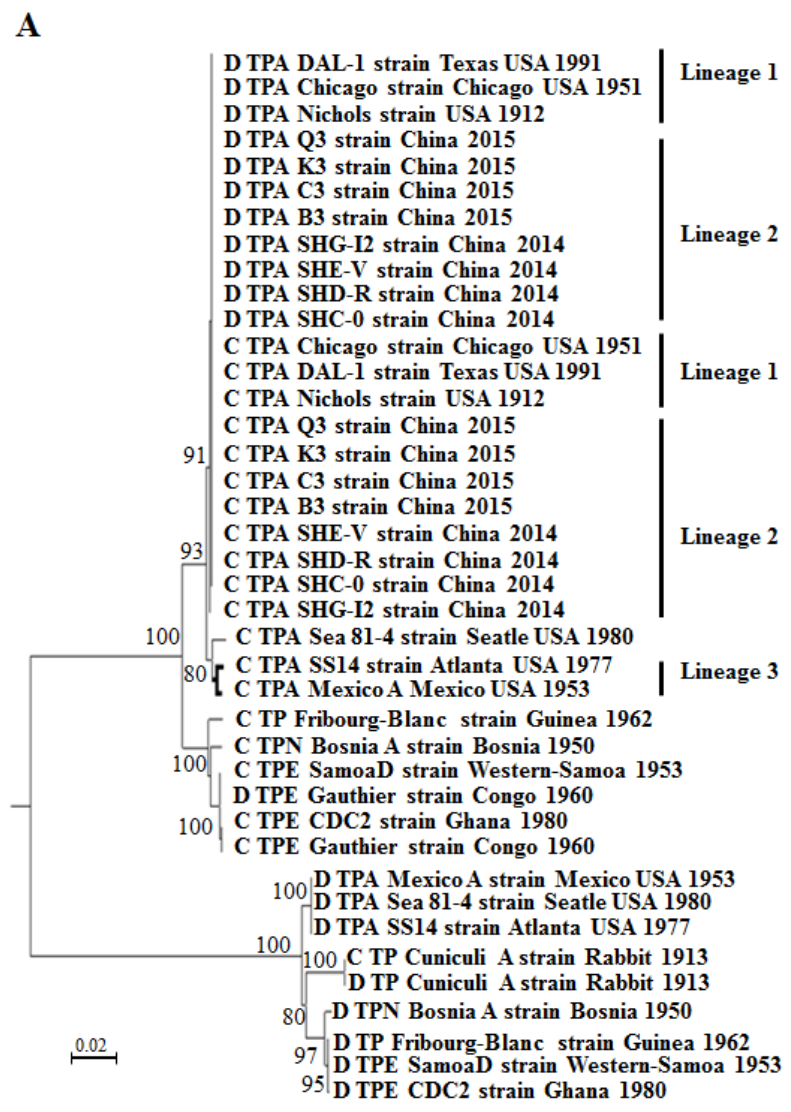

PCR primers are shown in Supplementary Table 4. The results confirmed $23(100 \%)$ of the 23 candidate SNVs (Supplementary Figure 2), suggesting that NGS is a highly sensitive and reliable method for identifying SNVs.

\section{Tracing the origin of Chinese TPA strains}

As most of the identified lineage-specific SNVs occurred in the tpr gene family, the tpr genes were used to further trace the origin of Chinese TPA strains. The $t p r$ gene family comprises 3 gene subfamilies and 12 paralogous genes: Subfamily I (tprC, D, I and F), Subfamily II (tprE, G, and J), and Subfamily III (tprA, $\mathrm{B}, \mathrm{H}, \mathrm{K}$, and $\mathrm{L}$ ). As potential outer membrane proteins and important virulence factors, the gene products from Subfamilies I and II exhibited conserved amino and carboxyl terminal regions and variable central regions, whereas Subfamily III presented highly variable regions across the full length of the genes [46, 47]. Due to the genetic characteristics of the various subfamilies of genes, both previous reports and the results of the present

$\mathbf{B}$

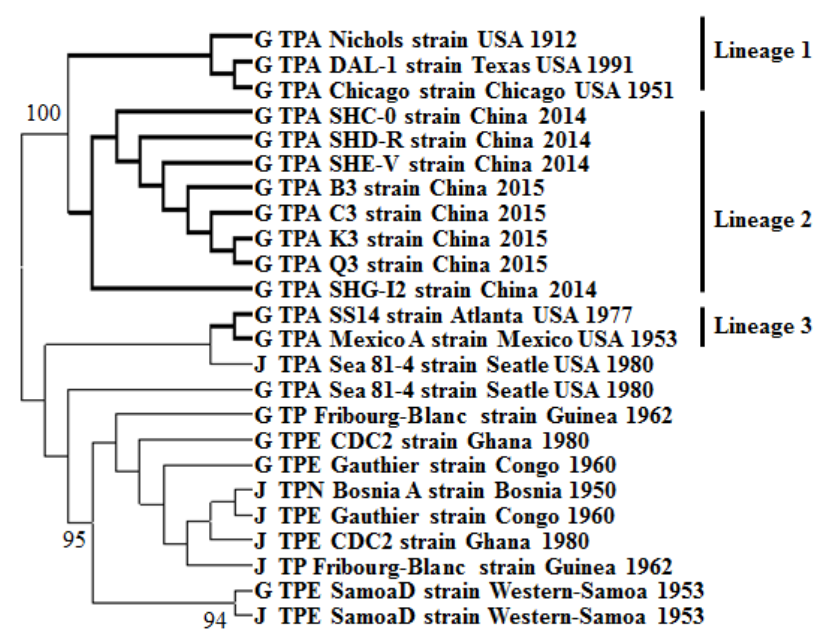

C

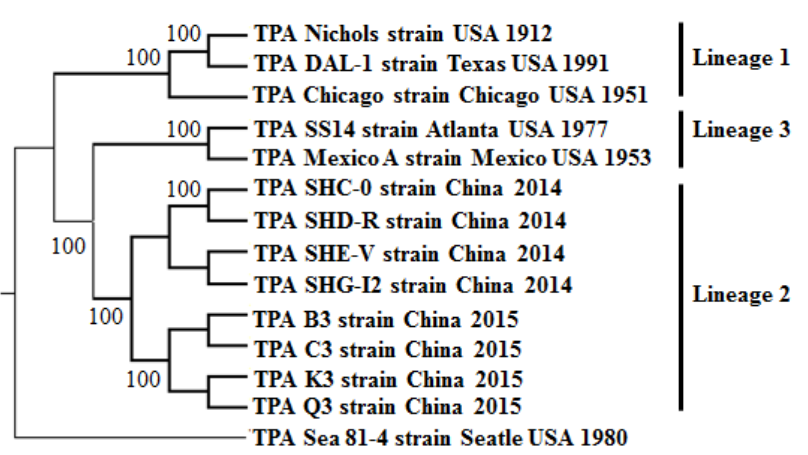

Figure 4: Phylogenetic analysis based on the tpr genes and SNVs of T. pallidum. A. Phylogenetic tree showing clusters of Subfamily I of $t p r$ genes. B. Phylogenetic tree showing clusters of Subfamily II of $t p r$ genes. C. Phylogenetic tree showing clusters of $t p r$ genes based on genomic SNVs after the removal of SNVs from the $\operatorname{tprD}$ and $\operatorname{tpr} G$ genes. The tree was constructed using MrBayes 3.1. Significant linkages using Bayesian phylogenetic inference analysis were considered as probabilities of $100 \%$. 
work indicated that the members of Subfamilies I and II are more suitable for the analysis of genetic evolution and might form the same species-specific cluster in a phylogenetic tree as did genomic SNVs (Supplementary Figures 3 and 4), whereas the Subfamily III members never cluster in a species-specific manner [11].

In the phylogenetic analysis of Subfamily I genes, three clades were presented with a $99 \%$ bootstrap value: Clade I included all of the $\operatorname{tpr} \mathrm{C}$ genes and some of the tprD genes (mainly from TPA Lineages 1 and 2); Clade II included the tprD genes from TPA strains (SS14, Mexico A and Sea 81-4) and TPE strains; and Clade III included the $t p r \mathrm{~F}$ and $t p r I$ genes (Supplementary Figure 3). There were three clusters in Clade I: all of the $t p r C$ and $t p r D$ genes from TPA Lineage 1 and Lineage 2 strains formed Cluster I with a $91 \%$ bootstrap value, while those from the Lineage 3 strains and Sea81-4 formed Cluster II with an $80 \%$ bootstrap value. These two clusters formed one large TPA cluster with a $93 \%$ bootstrap value. Outside this TPA cluster, the tprC genes from TPE and TEN strains formed Cluster III with a $100 \%$ bootstrap value, suggesting that the $t_{p r C}$ genes of Chinese TPA strains are most likely derived from Lineage 1 rather than Lineage 3, although the latter also showed a close relationship with the $\operatorname{tprC}$ gene cluster of Lineage 1 and Lineage 2 strains (Figure 4A). Furthermore, the findings suggested that all of the tprC genes of treponemal species had the same origin and that gene conversion from tprC to $t p r \mathrm{D}$ might have occurred in TPA Lineage 1 and 2 strains. Only the tprD genes derived from Lineage 3 and the TPE and TPN strains were included in Clade II and formed a clade with a 100\% bootstrap value (Supplementary Figure 3, Figure 4A). In Clade III, the tprF and tprI genes from all of the TPA strains formed one cluster, whereas the $t p r \mathrm{~F}$ and $t p r \mathrm{I}$ genes from TPE and TPN strains formed another cluster (Supplementary Figure 3).
In the phylogenetic analysis of Subfamily II genes, there were four clusters with high bootstrap values: all of the tprG genes and TPE and TPN-derived tprJ genes formed Cluster I; the T. paraluiscuniculi strain-derived tprE, tprG and tprJ genes formed Cluster II; all of the TPA-derived tprJ genes formed independent Cluster III; and all of the tprE genes derived from treponemes (except for the T. paraluiscuniculi strain) formed Cluster IV (Supplementary Figure 4). As shown in Cluster I (Supplementary Figure 4), the tprG genes of the Chinese TPA strains were most likely derived from Lineage 1 rather than Lineage 3. Similar to the tprD genes, the $t p r \mathrm{G}$ genes of Lineage 3 showed high homology with the genes of TPE and TPN strains, as shown in Cluster II (Figure 4B). Gene conversions were indicated between the tprJ and $t p r G$ genes in the TPE and TPN strains and the TPA Sea81-4 strain in addition to the $t p r \mathrm{E}, t p r \mathrm{G}$ and $t p r \mathrm{~J}$ genes from T. paraluiscuniculi strains. Because the tprG genes constituted the majority of Cluster I, tprG might have been the donor gene for gene conversion. Moreover, the $t p r \mathrm{E}$ genes were highly conserved among members of the genus Treponema, whereas the tprJ genes were highly conserved in TPA strains (except in Sea81-4).

Intragenomic gene conversion might have occurred between the $t p r \mathrm{C}$ and $t p r \mathrm{D}$ genes of Lineage 2 (Chinese TPA strains) and Lineage 1 (Figure 4A) and between the $t p r \mathrm{~F}$ and $t p r \mathrm{I}$ genes and $t p r \mathrm{G}$ and $t p r \mathrm{~J}$ genes of TPE and TPN strains (Supplementary Figure 3), as confirmed by the results of the present study and those of previous reports [36]. Additionally, gene conversion between $\operatorname{tprC}$ and tprD genes was identified in the TPE Gauthier strain. In addition, the high homology of the tprD and tprG genes between TPA Lineage 3 and the TPE and TPN strains suggested a close genetic linkage between these two species at the beginning of treponemal evolution. As noted above, although many more SNVs were shared between
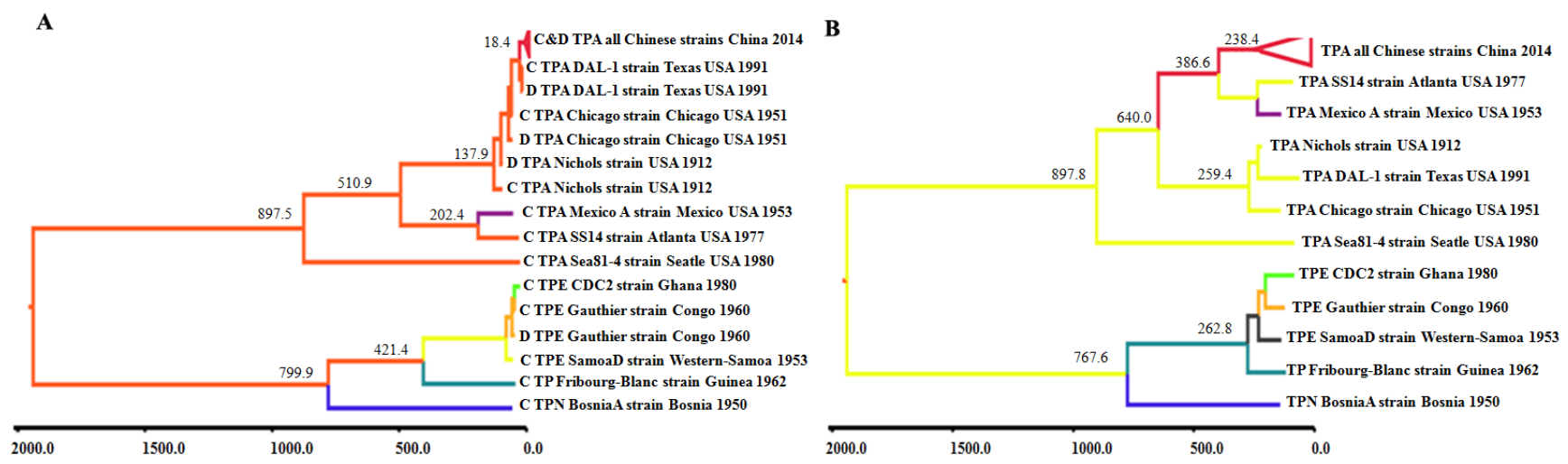

Figure 5: Maximum clade credibility trees of tpr family gene sequences and modified SNVs of T. pallidum. Phylogeographical trees were constructed using BEAST V1.6.2. The tree branches are colored according to their respective cluster or branch. The tMRCA of each cluster is labeled at the tree nodes. A. Phylogeographical analysis showing the clusters based on the $\operatorname{tp} C$ and $\operatorname{tpr} D$ gene sequences among treponemal strains. B. Phylogeographical analysis showing clusters based on the genomic SNVs of tpr family genes after removing the SNVs of $\operatorname{tpr} D$ and $\operatorname{tpr} G$ genes. tMRCA stands for time to the most recent common ancestor. 
TPA Lineage 2 and Lineage 3 than between Lineage 2 and Lineage 1 (268 vs. 0), Lineage 2 displayed a closer genetic relationship with Lineage 1 than with Lineage 3 in the genomic SNV-based ML tree, likely reflecting the close genetic relationship between Lineage 3 and the TPE and TPN strains. To address this concern, after these SNVs were removed from the $t p r \mathrm{D}$ and $t p r \mathrm{G}$ genes, the modified genomic SNVs were used to reconstruct an ML tree. As expected, three lineages were observed in the TPA strain clade, although Lineage 2 and Lineage 3 displayed a closer genetic relationship, forming one large cluster with high confidence (Figure 4C). These results suggest that Lineage 2 exhibited a mosaic genomic structure, involving the insertion of Lineage 1-derived genes (mainly tpr genes) into the Lineage 3-derived genomic backbone, further suggesting that Lineage 2 might have been the product of lateral gene transfer or recombination between Lineage 1 and Lineage 3.

To trace the origin of Chinese TPA strains and to estimate the timeline of global TPA strains, phylogeographical analyses were performed on the tprC and tprD genes from Clade I of Subfamily I using the BEAST v1.7.2 software package. As indicated in Figure 5A, the TPA and TPE and TPN strains formed an independent clade during their genetic evolution, and the same three lineages were identified as in the ML tree. Notably, the tMRCA of the TPA strains occurred approximately 897 (range 465-1363) years ago, while that of the TPE and TPN strains occurred approximately 799 (range 446-1178) years ago, suggesting that the TPA strains originated earlier than did the TPE and TPN strains. The tprC gene appeared earlier in evolution than did the tpr $\mathrm{D}$ gene in two independent clusters (TPA cluster and TPE and TPN cluster), suggesting that the tprC gene was most likely the donor of the $t p r \mathrm{D}$ gene via gene conversion. Within the TPA clade, Sea81-4 represents the earliest TPA isolate, and the cluster formed by the three TPA lineages might represent the most recent ancestor strain for the global outbreak of TPA: the timing of this ancestor was estimated at approximately 510 years ago, near the time when syphilis infections were first reported in Europe. Three current TPA lineages appeared sequentially in the timeline: Lineage 3 first appeared approximately 202 (98314) years ago, Lineage 1 appeared approximately 137 (67-215) years ago, and the Chinese TPA strains appeared approximately 18 (9-28) years ago.

Considering the distinct genetic linkages of Chinese TPA strains with Lineage 1 and Lineage 3 in various genomic regions, we further performed a phylogeographical analysis on modified SNV alignments (removing SNVs in $t p r \mathrm{D}$ and G genes). As expected, Lineage 2 and Lineage 3 exhibited a close relationship and shared the most recent common ancestor, whereas Lineage 2 displayed a much earlier tMRCA for modified genomic SNVs than for the tprC genes (238 vs. 18.4 years ago, Figure 5B), further establishing that Lineage 2 originated from multiple distinct parental strains. Although different tMRCAs of the three TPA lineages were estimated for genomic SNVs in contrast to the tprC gene, the order of the timeline of these lineages was the same in these two Markov chain Monte Carlo (MCMC) trees, with Sea814 representing the earliest TPA isolate and with three lineages appearing sequentially in various timelines: Lineage 3 the earliest, Lineage 1 the second earliest, and Lineage 2 the most recent. Similar to the timeline for the tprC and D genes, the tMRCA for the TPA strains in the genomic SNV-based MCMC tree occurred approximately 897 years ago, while the tMRCA for the TPE and TPN strains occurred approximately 767 years ago, further confirming that the TPA strains originated earlier than did the TPE and TPN strains. Overall, both the tpr gene and genomic SNV-based phylogeographical analyses firmly support the distinctive evolutionary characteristics of various treponemal species and clearly estimate the genesis and tMRCAs of treponemal species.

\section{DISCUSSION}

The first characterized genetic diversity of Chinese syphilis shows the utility of the NGS strategy for performing comparative genomic analysis of treponemal strains. To our knowledge, this is the first effort to sequence the entire TPA genome in China. Although it is widely accepted that TPA strains share high genetic homology, we revealed a distinct distribution pattern of SNVs in various TPA strains. In contrast to the Nichols strain, all of the Chinese TPA strains displayed an intermediate degree of variation (243-286 sites) in terms of genomic SNVs, while the Chicago and DAL-1 strains displayed minimal variation ( $<50$ sites) and the Mexico A, SS14 and Sea81-4 strains displayed maximal variation ( $>300$ sites). In this context, three independent TPA lineages were first identified through both SNV patterns and a phylogenetic analysis, and the strains within the lineage showed high similarity regarding SNV variation; e.g., the Chinese TPA strains shared more than 70\% (236 of 329) of their SNVs. Notably, the majority of the SNVs identified in TPA strains consisted of nonsynonymous sites, which likely altered the encoded amino acids. Moreover, most lineage-specific SNVs occurred in tpr genes and some hypothetical genes. Although little is known about the function of these hypothetical proteins, the high frequency of SNVs observed in some hypothetical proteins is important for pathogenesis or immune escape, which deserves further study. Therefore, many lineage-specific SNVs not only reflect the imprint of genetic evolution but also affect the function of genes and even the biological characteristics of TPA strains.

In the Chinese TPA strains, most lineage-specific SNVs occurred in $t p r$ genes and some hypothetical genes. Although little is known regarding the function of these hypothetical proteins, the high frequency of SNVs 
observed in some hypothetical proteins is important for pathogenesis or immune escape, which deserves further study. Tpr proteins play a critical role in the binding and recognition activity of TPA strains and are widely recognized by the host immune system [48, 49]. Thus, scientists have made great efforts in recent years to develop a tpr-gene-based vaccine against TPA infection [49].

Although penicillin therapeutic regimens have been highly effective for syphilis in the past half century, concerns have been raised that penicillin may be inadequate because "relapse" often occurs after penicillin therapy [40-44]. The lack of evidence to reasonably explain this treatment failure has plagued doctors for a long period of time. Nonsynonymous SNVs were first identified in the gene family of penicillin regulatory proteins in Chinese TPA strains (TPANIC_0500, TPANIC_0760, and TPANIC_0705) and Lineage 3 strains (TPANIC 0500). Homology modeling and SIFT analysis revealed that only one SNV (A825069T; I415F in the PBP3 protein) had a deleterious effect on the structural flexibility and the binding constant for substrate stability, as this SNV might be associated with penicillin resistance in T. pallidum (Figure 3A). Our findings in this study might partially explain the doctors' concerns, although additional studies are needed to confirm this hypothesis.

The phylogenetic analysis revealed that SNVs, gene conversion and lateral gene transfer constituted the main forces driving the evolution of TPA strains. In 2006, Gray et al. investigated the evolution of the $t p r$ gene among various subspecies and proposed that gene conversion has played a critical role in the evolution of Treponema [36]. In the present study, our phylogenetic analysis of tpr gene Subfamilies I and II not only confirmed this hypothesis but also further delineated the contribution of $t p r$ gene conversion to the dynamic evolution of TPA strains, as is showed in TPANIC_0136 and TPANIC_0548genes (Supplementary Figure 5 and 6). The intra-genomic gene conversion between $t p r C$ and tprD genes in TPA Lineage 1 and Lineage 2 might be responsible for the distinct evolution of Lineage 1 and Lineage 2 and might have led to the close genetic relationship between these two TPA lineages. In addition, common gene conversions between $t p r \mathrm{~J}$ and $t p r \mathrm{G}$ genes in all of the TPA strains except for the Sea81-4 strain might have contributed to the development of Lineage 3, Lineage 2 and Lineage 1. In addition, common gene conversions between the tprG and tprJ genes in the TPA Sea81-4 strain and TPE and TPN strains indicated a close genetic lineage between the TPA and TPE and TPN strains, suggesting that the Sea814 strain might represent the earliest TPA strain. Moreover, the close genetic relationship observed between the TPA Lineage 3 and TPE and TPN strains for the tprD and $t p r \mathrm{G}$ genes also suggests that Lineage 2 was the second earliest strain. Notably, Lineage 2 showed a closer relationship with Lineage 1 than with Lineage 3 in the $\operatorname{tprC}$ and
tprG gene-based phylogenetic tree, while Lineage 1 shared the maximum number of SNVs with Lineage 3. Thus, Lineage 1 was further identified as having a mosaic genomic structure, involving a Lineage-3-derived genomic backbone with the insertion of several Lineage1-derived tpr genes. Collectively, the recently reemerged Chinese TPA strains might reflect gene mutations and gene recombination events between existing TPA strains. China announced the extinction of syphilis in the 1960s; however, a new outbreak of syphilis was reported in 1996. Since then, syphilis has become a major public health problem [7, 8]. Because a syphilis outbreak occurred throughout almost the entire country, tracing the origin and understanding the genetic linkage of Chinese TPA strains with global TPA strains is critical for designing rational prevention measures.

Indeed, although epidemics of syphilis or the TPA strain were observed more than 500 years ago, both the phylogenetic relationship among Treponema and the evolutionary history of TPA strains remain controversial. In 1961, Cockburn TA proposed that pinta was the original form, which subsequently evolved into yaws, followed by endemic syphilis and ultimately venereal syphilis [48]. However, in 1965, Hudson suggested that venereal syphilis, endemic syphilis, yaws, and pinta were not in fact distinct diseases, but rather, the same disease with various manifestations [49]. In 2005, Armelagos et al. indicated the lack of a molecular distinction among these subspecies [52]. In addition, Gray et al. suggested that the TPA strain was not later in origin than the TPE strain, whereas Harper et al. recently suggested that the TPE and TPN strains exhibited an earlier origin than that of the TPA strain [32]. A review of these studies revealed a deficiency in summarizing the dynamics of treponemal evolution and global genetic variation in the treponemal genome, which might have provided insufficient evidence and led to incomplete conclusions. In the present study, the phylogeographical analyses of both the tprC and D genes and genomic SNVs clearly estimated the timeline for the genus Treponema and TPA strains.

During the first evolutionary phase of the genus Treponema, the TPA and TPE and TPN subspecies separated in genetic evolution and formed early ancestors in different timelines (879 and 799 years ago, respectively). Several scenarios have been proposed for the origin of the TPA species $[31,51]$. New World versus Old World hypotheses concerning the origin of venereal syphilis has also long been debated. The results of the present study suggest that TPA and TPE and TPN strains shared a common ancestor and had the same origin. Although the Sea81-4 strain was only isolated in 1980 and showed the highest genetic homology with the TPE and TPN strains, strain Sea81-4 mostly closely resembled the earliest ancestor of TPA in genetic evolution, which not only further supports the common genesis of various treponemal species but also suggests that TPA strains 
originated in North America.

The global epidemic of treponemes initiated the second evolutionary phase. Similarly, the most recent common ancestor of global epidemic TPA strains occurred earlier than did that of TPE and TPN strains (510 vs. 421 years ago). The appearance of the most recent TPA ancestor suggests the acquisition of some abilities of TPA strains at this time point, which has been attributed to significant genetic variation in pathogens (such as gene conversion in the $t p r \mathrm{G}$ and $t p r \mathrm{~J}$ genes). In this context, significant genetic alterations in pathogens are typically accompanied by a switch from an old host environment to a new host population, suggesting that the earliest introduction of TPA strains occurred from North America into Europe.

Considering that Europe initiated the global transmission of TPA strains during the $16^{\text {th }}$ century [52, 53], TPA strains subsequently evolved into two distinctive lineages. In addition, accompanied by the prevalence of colonialism, frequent global distribution might have led to multiple introductions of TPA strains to a single continent, suggesting that the common ancestor of Lineage 2 and Lineage 3 originated approximately 386 years ago and that the most common ancestor of Lineage 1 and Lineage 2 originated approximately 138 years ago. During this phase, TPA strains gradually lost the high genetic similarity of the tpr gene family with TPE and TPN strains.

In 1504, a Chinese doctor in Guangzhou described a syphilis-like manifestation corresponding to the earliest opening of a port to Western people [54]. The syphilis epidemic in China had been eradicated through endeavors of the Chinese government from 1958 to 1970 [5]. The reemergence of syphilis/TPA in China in 1990 might reflect recombinant events mediated through recently introduced Lineage 1 and previously existing Lineage 3 . Indeed, the phylogenetic analysis of $t p r$ genes and modified SNVs provided the following evidence: 1) the Chinese TPA strains showed a closer genetic linkage of $t p r \mathrm{C}, t p r \mathrm{D}$ and ${ }_{t p r} \mathrm{G}$ genes with Lineage 1 than with Lineage 3;2) the Chinese TPA strains exhibited a close genetic linkage of the majority of genes with Lineage 3; and 3) gene conversions occurred in $t p r \mathrm{C}$ and $t p r \mathrm{D}$ genes from TPA Lineage 1 and Lineage 2 strains and in $t p r \mathrm{G}$ and $t p r \mathrm{~J}$ genes from TPE and TPN strains. The tMRCA of Lineage 2, as estimated in the $\operatorname{tpr} \mathrm{C} / \mathrm{D}$ genes, occurred approximately 18 years ago, providing further evidence of the reemergence of TPA strains in China. Notably, the newly emerged TPA Lineage 2 strain will cause a new wave of global transmission, which will also require serious measures to strengthen the global monitoring of the TPA epidemic.

Taken together, genomic SNVs and tpr-genebased phylogenetic and phylogeographical analyses not only revealed the emergence of Chinese TPA strains as a new lineage but also clearly delineated the origin and evolutionary process of TPA subspecies during global transmission. However, there are some limitations in this study: 1) the absence of early TPA genome sequences in the phylogeographical analysis might lead to an overly wide range being estimated for the origin of TPA strains and lineages; 2) the absence of European-derived TPA genome sequences might make it difficult to determine the origin of the European TPA epidemic; 3) the absence of Chinese TPA isolates obtained before the 1960s may be an obstacle to tracing the exact ancestor of Chinese TPA strains; and 4) due to the high cost of NGS and of the isolation of TPA strains, only eight genomes of Chinese TPA isolates were investigated here, and their representativeness of common Chinese TPA strains must be studied further in the future.

\section{MATERIALS AND METHODS}

\section{Sample collection}

In this study, we collected T. pallidum from the skin lesions of eight patients suffering from early syphilis according to diagnostic criteria [55], including one primary syphilis patient (SHC-0), three secondary syphilis patients with central nervous system (CNS) involvement (SHD-R, SHE-V, Q3) and four secondary syphilis patients (SHG-I2, B3, C3, and K3) (Supplementary Table 1). Written informed consent was obtained from all of the patients. The protocol for investigating human and animal tissues was reviewed and approved by the Ethics Committee of the Shanghai Skin Disease Hospital. New Zealand white rabbits were used for the isolation and propagation of the TPA strain. Animal care was provided in accordance with the procedures outlined in the Guide for the Care and Use of Laboratory Animals under the protocols approved by the Animal Care and Use Committee of the Shanghai Skin Disease Hospital.

\section{TPA strain isolation and harvest}

The isolation and propagation from patients of TPA strains followed a previously described protocol [56]. Briefly, male seronegative New Zealand white rabbits were infected via injected perianal condyloma or chancre exudate into each testicle. During infection, rabbit serum was collected and used for Treponema pallidum particle assay (TPPA) and rapid plasma regain test (RPR) every two days. After the development of a firm orchitis, positive TPPA and positive RPR within 30-40 days, the animals were euthanized via the intracardiac injection of sodium phenobarbital, and the testes were removed aseptically. To harvest TPA, the rabbit testicular tissues were dissected out and squeezed several times in heat-inactivated normal rabbit serum (NRS). After being centrifuged at 1,000 $\mathrm{x} g$ for $10 \mathrm{~min}$, supernatant containing treponemal cells was carefully collected and centrifuged again at $14,100 \mathrm{x} g$ for 
3 min. The resulting pellet containing treponemal cells was washed twice in PBS buffer and centrifuged at 14,100 $\mathrm{x} g$ for $3 \mathrm{~min}$. The resulting treponemal suspension was aliquoted into 10 vials, (1 mL in each vial), subsequently confirmed by dark-field microscopy and stored in liquid nitrogen for further use. All of the sequencing samples were from the first passage in rabbits.

\section{DNA extraction}

The bacterial DNA was then extracted from a frozen treponemal suspension using the QIAGEN Genomic-tip 100/G kit (Qiagen Inc., Chatsworth, CA) according to the manufacturer's instructions, and the DNA samples were stored at $-20^{\circ} \mathrm{C}$ until use.

\section{Library construction and next-generation sequencing}

DNA libraries were constructed with genomic DNA extracted using the CTAB method (Illumina, CA, USA) according to the manufacturer's instructions. PE (pairedend) libraries with an insert size of $300 \mathrm{bp}$ were prepared for each sample. The methods of DNA manipulation, including the generation of single-molecule arrays, cluster growth and paired-end sequencing, were performed using a HiSeq 2500 sequencer (Illumina, CA, USA) with the default parameters according to standard protocols. The Illumina base-calling pipeline was employed to process raw fluorescent images and call sequences. Raw reads of low quality obtained from paired-end sequencing (those with consecutive bases covered by fewer than five reads) were discarded. All of the sequence data (fastq) were deposited in the NCBI SRA database under project number PRJNA305961.

\section{Data analysis and SNV annotation}

Sequence reads were purified using the FASTX toolkit (http://hannonlab.cshl.edu/fastx_toolkit/). After adaptor trimming and quality trimming, the clean reads were mapped to the Nichols reference genome (GenBank accession: CP004010.2) using Bowtie2 with the very-fastlocal parameter. The parameter values were as follows: max 0 mismatch and 0 gap in a seed alignment, excluding the reads after 5 failed attempts to extend a sequence. Other parameters were set as default parameters. SNVs from the alignments were called using SAMTools [57] and output in pileup format. To validate the resulting nonredundant candidate SNVs in eight sequenced genomes, the numbers of the most abundant (n1) and second most abundant (n2) nucleotides at each SNV in each strain (according to the number of reads in each strain supporting the presence of the nucleotide) were examined. High- quality SNVs satisfied the following criteria: (i) the most abundant base was different from that in the Nichols genome; (ii) $n 1+n 2 \geq 10$; and (iii) $n 1 /(n 1+n 2) \geq 0.7$.

\section{Phylogenetic and phylogeographical analyses of treponemal strains based on genomic SNVs and tpr genes}

To investigate the genetic diversity of Chinese TPA strains, a total of 329 SNVs in the Nichols strain were collected and used for phylogenetic analysis. Phylogenetic trees of genomic SNVs were reconstructed through a maximum likelihood (ML) heuristic search in phylogenetic analysis using parsimony (PAUP) v4.0 b10, and the general time-reversible model with a proportion of invariant sites and a gamma distribution $(\mathrm{GTR}+\mathrm{\gamma}+\mathrm{R})$ was selected as the most appropriate analysis model using ModelTest software [58]. To generate the maximumlikelihood tree, the statistical robustness of the tree and the reliability of the branching patterns were confirmed through bootstrapping tests for 1,000 replicates, and the effective phylogenetic relationship was supported by a $>80 \%$ bootstrap value. To confirm the genetic linkage as indicated by the ML tree, a Bayesian inferred tree was also reconstructed using MrBayes 3.1 as previously described [34]. To fully elucidate the origin of Chinese-derived TPA strains, additional phylogenetic analyses of both the tpr genes and genomic SNVs were performed using PAUP 4.0 software, and phylogeographical analyses were conducted using the BEAST v1.6.2 software package according to the manufacturer's instructions [59]. Each MCMC analysis was run for at least 50 million generations and sampled every 10,000 generations in BEAST V1.6.2. The program Tracer V1.5.1 (tree.bio.ed.ac.uk/software/tracer/) was employed to determine convergence and to determine whether the effective sample size (ESS) was $>200$. When the ESS was $<200$, the MCMC chain length was elongated to 100 million. To construct maximum clade credibility (MCC) trees, the initial $25 \%$ of trees generated were discarded as burn-in, and the living trees per run were summarized using Tree Annotator, as implemented in BEAST V1.6.2. All of the trees were assessed and edited using FigTree V1.3.1 (tree.bio.ed.ac.uk/software/ figtree/), which was also employed to estimate the time of the most recent common ancestor (tMRCA) of various nodes on the MCC tree. Posterior probabilities for the internal nodes were calculated from the posterior density of the trees. The statistical uncertainty in parameter estimates was reflected based on the values of the $95 \%$ highest posterior density (HPD) credible region (CR). 


\section{SIFT analysis and three-dimensional (3D) protein- folding structure prediction}

The SIFT algorithm was applied to predict SNVs that are likely to affect functions of penicillin regulatory protein. Briefly, SIFT identifies homologs of the gene of interest in other bacteria by 1) scoring the conservation of the positions where mutations are detected and 2) weighting this score according to the nature of the amino acid change. These measures are incorporated into a normalized probability score, and a SIFT score $\leq 0.05$ indicates a predicted functional impact. The recommended $>3.5$ conservation threshold was used to filter biased predictions. All of the mutated penicillin regulatory genes found in TPA strains were used for SIFT analysis. Predictions of SNVs that destabilize the protein structure were obtained using the Protein Homology/analog Y Recognition Engine V 2.0 [60].

\section{PCR-based Sanger sequencing}

We selected 23 candidate SNV sites and their flanking sequences, including $6 \mathrm{SNVs}$ in the PBP gene, $7 \mathrm{SNVs}$ in the tpr gene and $10 \mathrm{SNVs}$ in other genes. The PCR primers were designed using Primer 5.0 software to fall within $100 \mathrm{nt}$ upstream or downstream of the position of SNV and were used to amplify these regions by PCR. The PCR product preparation and Sanger sequencing were performed by Life Technologies. Sanger sequencing was performed using the BigDye Terminator v1.1 Cycle Sequencing Kit. After ethanol purification, the PCR products were run on an ABI 3730 sequencer. The resulting data were screened for mutations through BioEdit software alignment.

\section{ACKNOWLEDGMENTS}

We gratefully acknowledge support from the National Natural Science Foundation of China (81371862, 81572039), the Shanghai Commission for Science and Technology (15JC1403000 and 15431902900), the Chinese National Key Program on Basic Research (2014CB965002), the Shanghai Health and Family Planning Commission (2013SY068), Program of Shenzhen Science Technology and Innovation Committee (JCYJ20130329171031740, CXZZ20130515163643 and JCYJ20120831144704366).

\section{CONFLICTS OF INTEREST}

There is no conflict of interest.

\section{Author's contributions}

P.Y.Z. and J.H. conceived and designed the experiments. H.J.Z., Y.Z.W. and J.H. performed sequencing and analyzed the sequencing data. K.Q.W., B.L., S.F.Z. and Y.D.L. performed the animal experiments. J.S. performed the validation assay. Z.F.M. performed the evolution analysis. J.H. and P.Y.Z. contributed reagents/ materials/analysis tools. Z.F.M., J.S., J.H. and P.Y.Z. wrote the paper.

\section{REFERENCES}

1. Schaudin FR and Hoffmann E. VorläufigerBerichtüber das Vorkommen von Spirochaeten in syphilitischenKrandkheitprodukten und beiPapillomen. Arb KGesund.1905; 22:527-534.

2. Centers for Disease Control and Prevention. Sexually Transmitted Disease Surveillance 2013. Atlanta: U.S. Department of Health and Human Services. 2014.

3. Patton ME, Su JR, Nelson R, Weinstock H and Centers for Disease Control and Prevention (CDC). Primary and secondary syphilis--United States, 2005-2013.MMWR Morb Mortal Wkly Rep. 2014; 63:402-406.

4. StammLVandMudrak B. Old foes, new challenges: syphilis, cholera and TB. Future Microbiol.2013; 8:177-189.

5. Cohen MS, Henderson GE, Aiello PandZheng H. Successful eradication of sexually transmitted diseases in the People's Republic of China: implications for the 21 st century. J Infect Dis. 1996; 174Suppl 2: S223-229.

6. Chinese Center for Disease Prevention and Control \& Center for STI and AIDS.Prevention and Control 2011 report on syphilis and gonorrhea epidemic analysis in China. Bull STI Prev Control.2012; 26:9-19.

7. Dong GX, Yue XL, Teng F, Jiang N and Men PX. Syphilis in China from 2000 to 2013: epidemiological trends and characteristics. Chinese; Chinese Journal of Dermatology.2014; 47:310-315.

8. Chen ZQ, Zhang GC, Gong XD, Lin C, Gao X, Liang GJ, Yue XL, Chen XS and Cohen MS. Syphilis in China: results of a national surveillance programme. Lancet.2007; 369:132-138.

9. Dai T, Li K, Lu H, Gu X, Wang Q and Zhou P. Molecular typing of Treponema pallidum: a 5-year surveillance in Shanghai, China. JClinMicrobiol.2012; 50:3674-3677.

10. Marra C, Sahi S, Tantalo L, Godornes C, Reid T, Behets F, Rompalo A, Klausner JD, Yin Y, Mulcahy F, Golden MR, Centurion-Lara AandLukehart SA. Enhanced molecular typing of treponemapallidum: geographical distribution of strain types and association with neurosyphilis. J Infect Dis. 2010; 202:1380-1388.

11. Centurion-Lara A,Giacani L, Godornes C, Molini BJ, Brinck Reid TandLukehartSA.Fine analysis of genetic diversity of the tpr gene family among treponemal species, 
subspecies and strains.PLoSNegl Trop Dis.2013; 7:e2222.

12. MerkerM,Blin C, Mona S, Duforet-Frebourg N, Lecher S, Willery E, Blum MG, Rüsch-Gerdes S, Mokrousov I, Aleksic E, Allix-Béguec C, Antierens A, AugustynowiczKopeć E, et al. Evolutionary history and global spread of the Mycobacterium tuberculosis Beijing lineage. NatGenet.2015; 47:242-249.

13. PětrošováH,Pospíšilová $P$, Strouhal $M$, Čejková D, Zobaníková M, Mikalová L, Sodergren E, Weinstock GM and Šmajs D. Resequencing of Treponema pallidum ssp. pallidum strains Nichols and SS14: correction of sequencing errors resulted in increased separation of syphilis treponemesubclusters. PLoS One.2013; 8: e74319.

14. Fraser CM, Norris SJ, Weinstock GM, White O, Sutton GG, Dodson R, Gwinn M, Hickey EK, Clayton R, Ketchum KA, Sodergren E, Hardham JM, McLeod MP, et al. Complete genome sequence of Treponema pallidum, the syphilis spirochete. Science.1998; 281:375-388.

15. Zobaníková $\mathrm{M}$, Mikolka $\mathrm{P}$, Cejková $\mathrm{D}$, Pospíšilová $\mathrm{P}$, Chen L, Strouhal M, Qin X, Weinstock GMandSmajs D. Complete genome sequence of Treponema pallidum strain DAL-1.Stand. GenomicSci.2012; 7:12-21.

16. Pětrošová $\mathrm{H}$, Zobaníková $\mathrm{M}$, Čejková $\mathrm{D}$, Mikalová L, Pospíšilová P, Strouhal M, Chen L, Qin X, Muzny DM, Weinstock GMandŠmajs D. Whole genome sequence of Treponema pallidum ssp. pallidum, strain Mexico A, suggests recombination between yaws and syphilis strains. PLoSNegl Trop Dis. 2012; 6:e1832.

17. Giacani L, Iverson-Cabral SL, King JC, Molini BJ, Lukehart SA andCenturion-Lara A. Complete Genome Sequence of the Treponema pallidum subsp. pallidum Sea81-4 Strain. GenomeAnnounc.2014; 2:e00333-14.

18. Matejková P, Strouhal M, Smajs D, Norris SJ, Palzkill T, Petrosino JF, SodergrenE, Norton JE, Singh J, Richmond TA, Molla MN, Albert TJ and Weinstock GM. Complete genome sequence of Treponema pallidum ssp. pallidum strain SS14 determined with oligonucleotide arrays. BMCMicrobiol. 2008;8:76.

19. Giacani L, Jeffrey BM, Molini BJ, Le HT, Lukehart SA, Centurion-Lara AandRockeyDD.Complete genome sequence and annotation of the Treponema pallidum subsp. pallidum Chicago strain. J Bacteriol.2010; 192:2645-2646.

20. 20 Cejková D, Zobaníková M, Chen L, Pospíšilová P, Strouhal M, Qin X, Mikalová L, Norris SJ, Muzny DM, Gibbs RA, Fulton LL, Sodergren E, Weinstock GM, et al. Whole genome sequences of three Treponema pallidum ssp. pertenue strains: yaws and syphilis treponemes differ in less than $0.2 \%$ of the genome sequence. PLoSNegl Trop Dis.2012; 6:e1471.

21. Zobaníková M, Strouhal M, Mikalová L, Cejková D, Ambrožová L, Pospíšilová P, Fulton LL, Chen L, Sodergren E, Weinstock GMandSmajsD.Whole genome sequence of the Treponema Fribourg-Blanc: unspecified simian isolate is highly similar to the yaws subspecies. PLoSNeglTrop
Dis. 2013; 7:e2172.

22. Staudová B, Strouhal M, Zobaníková M, Cejková D, Fulton LL, Chen L, Giacani L, Centurion-Lara A, Bruisten SM, Sodergren E, Weinstock GMandSmajs D. Whole genome sequence of the Treponema pallidum subsp. endemicum strain Bosnia A: the genome is related to yaws treponemes but contains few loci similar to syphilis treponemes. PLoSNegl Trop Dis. 2014; 8:e3261.

23. Smajs D, Zobaníková M, Strouhal M, Čejková D, DuganRocha S, Pospíšilová P, Norris SJ, Albert T, Qin X, Hallsworth-Pepin K, Buhay C, Muzny DM, Chen L, et al. Complete genome sequence of Treponema paraluiscuniculi, strain Cuniculi A: the loss of infectivity to humans is associated with genome decay. PLoS One.2011; 6: e20415.

24. GiacaniLandLukehart SA. The endemic treponematoses. ClinMicrobiol Rev. 2014; 27:89-115.

25. Smajs D, Norris SJ and Weinstock GM. Genetic diversity in Treponema pallidum: implications for pathogenesis, evolution and molecular diagnostics of syphilis and yaws. Infect Genet Evol. 2012; 12:191-202.

26. Paster BJ.2010. Phylum XV.SpirochaetesGarrity and Holt 2001, p471-566. In: Krieg NR, Ludwig W, Whitman WB, Hedlund BP, Paster BJ, Staley JT, Ward N, Brown D and Parte, A(eds). Bergey's manual of systematic bacteriology. New York, NY, USA: Springer New York.

27. Miao RM andFieldsteelAH.Genetic relationship between Treponema pallidum and Treponema pertenue, two noncultivable human pathogens.J Bacteriol.1980; 141:427429.

28. Nechvátal L, Pětrošová H, Grillová L, Pospíšilová P, Mikalová L, Strnadel R, Kuklová I, Kojanová M, Kreidlová M, Vaňousová D, Procházka P, Zákoucká H, Krchňáková A, et al. Syphilis-causing strains belong to separate SS14like or Nichols-like groups as defined by multilocus analysis of 19 Treponema pallidum strains. Int. J Med Microbiol.2014; 304:645-653.

29. MikalováL,Strouhal M, Čejková D, Zobaníková M, Pospíšilová P, Norris SJ, Sodergren E, Weinstock GMandŠmajsD.Genome analysis of Treponema pallidum subsp. pallidum and subsp. pertenue strains: most of the genetic differences are localized in six regions. PLoS One.2010; 5:e15713.

30. Meyer C, Jung C, Kohl T, Poenicke A, Poppe A and Alt KW. Syphilis 2001--a palaeopathological reappraisal. Homo.2002; 53:39-58.

31. Brothwell D. North American treponematosis against the bigger world picture. In: Powell M and Cook D (eds). The myth of syphilis: the natural history of treponematosis in North America. Gainesville: University Press of Florida. 2005.pp. 480-496.

32. Harper KN, Ocampo PS, Steiner BM, George RW, Silverman MS, Bolotin S, Pillay A, Saunders NJandArmelagosGJ.On the origin of the treponematoses: a phylogenetic approach. PLoSNegl Trop Dis. 2008; 2:e148. 
33. Mays S, Crane-Kramer $\mathrm{G}$ andBayliss A. Two probable cases of treponemal disease of Medieval date from England. Am J PhysAnthropol.2003; 120:133-143.

34. Drummond AJ andRambaut A. BEAST: Bayesian evolutionary analysis by sampling trees. BMC Evol Biol. 2007; 7:214.

35. Sun ES, Molini BJ, Barrett LK, Centurion-Lara A, Lukehart SA and Van Voorhis WC. Subfamily I Treponema pallidum repeat protein family: sequence variation and immunity. Microbes Infect. 2004; 6:725-737.

36. Gray RR, Mulligan CJ, Molini BJ, Sun ES, Giacani L, Godornes C, Kitchen A, Lukehart SA and Centurion-Lara A. Molecular evolution of the tprC, D, I, K, G, and J genes in the pathogenic genus Treponema. MolBiolEvol.2006; 23:2220-2233.

37. Giacani L, Molini BJ, Kim EY, Godornes BC, Leader BT, Tantalo LC, Centurion-Lara AandLukehartSA.Antigenic variation in Treponema pallidum: TprK sequence diversity accumulates in response to immune pressure during experimental syphilis. J Immunol.2010; 184:3822-3829.

38. Zhou HQ, Ning LW, Zhang HX andGuo FB. Analysis of the relationship between genomic GC Content and patterns of base usage, codon usage and amino acid usage in prokaryotes: similar GC content adopts similar compositional frequencies regardless of the phylogenetic lineages. PLoS One.2014; 9:e107319.

39. Lightfield J, Fram NR and Ely B. Across bacterial phyla, distantly-related genomes with similar genomic GC content have similar patterns of amino acid usage. PLoS One.2011; 6:e17677.

40. Zhou P, Gu X, Lu H, Guan Z andQian Y. Re-evaluation of serological criteria for early syphilis treatment efficacy: progression to neurosyphilis despite therapy. Sex Transm Infect. 2012; 88:342-345.

41. Douglas JM Jr. Penicillin treatment of syphilis: clearing away the shadow on the land. JAMA.2009; 18:769-771.

42. Fowler VG Jr, Maxwell GL, Myers SA, Shea CR, Livengood CN 3rd, Prieto VG and Hicks CB.Failure of benzathine penicillin in a case of seronegative secondary syphilis in a patient with acquired immunodeficiency syndrome: case report and review of the literature. Arch Dermatol.2001; 137:1374-1376.

43. Rolfs RT, Joesoef MR, Hendershot EF, Rompalo AM, Augenbraun MH, Chiu M, Bolan G, Johnson SC, French P, Steen E, Radolf JD and Larsen S.A randomized trial of enhanced therapy for early syphilis in patients with and without human immunodeficiency virus infection. The Syphilis and HIV Study Group.NEngl J Med. 1997; 337:307-314.

44. Stapleton JT, Stamm LV andBassford PJ Jr. Potential for development of antibiotic resistance in pathogenic treponemes. Rev Infect Dis. 1985; 7(Supp12):S314-317.

45. Zapun A, Contreras-Martel C andVernet T. Penicillin- binding proteins and beta-lactam resistance. FEMS Microbiol Rev. 2008; 32:361-385.

46. Giacani L, Brandt SL, Puray-Chavez M, Reid TB, Godornes C, Molini BJ, Benzler M, Hartig JS, Lukehart SA and Centurion-Lara A.Comparative investigation of the genomic regions involved in antigenic variation of the TprK antigen among treponemal species, subspecies, and strains. J Bacteriol.2012; 194:4208-4225.

47. Centurion-Lara A, Godornes C, Castro C, Van Voorhis WC andLukehart SA. The tprK gene is heterogeneous among Treponema pallidum strains and has multiple alleles. Infect Immun. 2000; 68:824-831.

48. Cockburn TA. The origin of the treponematoses. Bull World Health Organ. 1961; 24:221-228.

49. Hudson EH. Treponematosis in perspective. Bull World Health Organ. 1965; 32:735-748.

50. Armelagos GJ, Brown PJ and Turner B. Evolutionary, historical and political economic perspectives on health and disease.SocSci Med. 2005; 61:755-765.

51. Baker BJ andArmelagos GJ. The origin and antiquity of syphilis: paleopathological diagnosis and interpretation. CurrAnthropol.1988; 29:703-738.

52. Fernandez de Oviedo y Valdes G. Sumario de la natural historia de lasIndias. Mexico: Fondo de CulturaEconomico. 1526.

53. Diaz de Isla R. Treatise on the serpentine malady, which in Spain is commonly called bubas, which was drawn up in the hospital of All Saints in Lisbon. 1539.

54. $\mathrm{Hu} \mathrm{CK}, \mathrm{Ge} \mathrm{Y}$ and Chen ST. Control and eradication of syphilis in China. Beijing Sci Conference.1964:167-177

55. Li K, Wang $\mathrm{C}, \mathrm{Lu} \mathrm{H}, \mathrm{Gu} \mathrm{X}$, Guan Z and Zhou P. Regulatory $\mathrm{T}$ cells in peripheral blood and cerebrospinal fluid of syphilis patients with and without neurological involvement. PLoSNeglTrop Dis. 2013; 7:e2528.

56. Lukehart SA and Marra CM. Isolation and laboratory maintenance of Treponema pallidum. CurrProtocMicrobiol.2007; Chapter 12:Unit 12A.1.

57. Li H, Handsaker B, Wysoker A, Fennell T, Ruan J, Homer N, Marth G, Abecasis G, Durbin R; 1000 Genome Project Data Processing Subgroup. The Sequence Alignment/Map format and SAMtools.Bioinformatics. 2009; 25:2078-2079.

58. Posada D and Buckley TR. Model selection and model averaging in phylogenetics: advantages of the AIC and Bayesian approaches over likelihood ratio tests. Syst Biol. 2004; 53:793-808.

59. Lemey P, Suchard M and Rambaut A. Reconstructing the initial global spread of a human influenza pandemic: a Bayesian spatial-temporal model for the global spread of H1N1pdm. PLoS. 2009; Curr.1: RRN1031.

60. Kelley LA, Mezulis S, Yates CM, Wass MN and Sternberg MJ. The Phyre2 web portal for protein modeling, prediction and analysis.Nat Protocols.2015; 10:845-858. 